\title{
Effect of critical care pharmacist on antibiotic medication optimizations, reduce drug costs but not increase mortality: A retrospective cohort study in the real world research
}

\section{Hongyan Gu}

Beijing Shijitan Hospital https://orcid.org/0000-0003-3769-9233

Lulu Sun

Capital Medical University Affiliated Beijing Shijitan Hospital

\section{Bo Sheng}

Capital Medical University Affiliated Beijing Shijitan Hospital

\section{Xuyun Gu}

Capital Medical University Affiliated Beijing Shijitan Hospital

\section{Suozhu Wang}

Capital Medical University Affiliated Beijing Shijitan Hospital

Wei Chen ( $\sim$ antiinfectious@163.com )

Capital Medical University https://orcid.org/0000-0003-3013-2832

\section{Research article}

Keywords: Critical care, Interventions, Critical care pharmacist, Optimizations, Mortality, Charges

Posted Date: August 13th, 2020

DOI: https://doi.org/10.21203/rs.3.rs-55961/v1

License: (c) (i) This work is licensed under a Creative Commons Attribution 4.0 International License.

Read Full License 


\section{Abstract \\ Background}

We aims to investigate the roles of clinical pharmacist on optimizing the antibiotic pharmacotherapy regimens and achieved better clinical and economic outcomes in the critical care unit (ICU).

\section{Methods}

A retrospective cohort study in real world was undertaken from the year of 2016 to 2017 as the pharmacist pre-intervention period and 2018 to 2019 as the pharmacist intervention period in ICU. All interventions and consensus with clinicians were recorded. The outcomes of the patients' mortality, microorganism detections, antibiotic utilities, length of ICU stay (LOS), costs of the antibiotics and the total drugs used were reviewed.

\section{Results}

Of 1436 patients were evaluated and 1252 recommendations were identified. The main points of the pharmacist interventions were medication regimen adjustments (52.32\%) and drug withdrawal (22.60\%). Before and after the pharmaceutical interventions, the AUD of all antimicrobials consumed decreased from 211.83 to $174.02(p=0.000)$, the rate of antimicrobial utility decreased from $89.88-86.82 \%(p=$ $0.001)$, mortality reduced from $18.73-15.21 \%(p=0.002)$, antibiotic charges were from $\varangle 8,644 \pm 12,556$ to $\varangle 5,587 \pm 7,606(p=0.000)$ with $39 \%$ reduction.

\section{Conclusions}

The services provided by the clinical pharmacist with highly professional training could optimize the antibiotic therapy regimes, saved the drug costs and did not increase mortalities.

\section{Background}

Infection is frequent among patients in the intensive care unit (ICU). Antibiotics are one of the most common treatment options administered in the ICU[1, 2]. The significant differences between the patients in ICU and general wards were multiple drugs co-administrations, limited physiological reserve, varies of complications and hemodynamic alterations increased the variations of drug pharmacokinetics and pharmacodynamics (PK/PD) in vivo[3, 4], which means that the dosage, dosing frequency, delivery route, duration of antibiotics or drug adverse reaction (ADR) occurrence rates of the antibiotics prescribed to the ICU patients may be totally different from that of the patients in the general ward[4]. All of these factors increase the difficulty of prescribing in the ICU and the rate of suboptimal prescribing may be even greater than the general infectious patients. 
Clinical roles of pharmacists in the medical and health care settings has not been fully described. In recent years, several studies improved the positive influence of clinical pharmacists on the health care of patients with chronic diseases such as cardiovascular diseases, diabetes and anticoagulant therapy[57]. Care of the critical patients has become a multidiscipline[8] and antimicrobial stewardship (AMS) programs have been developed for optimizing the treatment of infections, to reduce infection-related morbidity and mortality $[9,10]$. Participation of a critical care pharmacist resulted in the identification and treatment of multiple medication-related problems[11], which improved clinical and economic outcomes[8, 12-14].

In China, it is currently impossible to assign one clinical pharmacist to each ICU in the large medical institutions, and the role of the critical care pharmacist was also not fully demonstrated. We conducted an intervention study to explore the associations between the provision of clinical pharmacy services in critically ill patients applied with anti-infectious medication therapy and the major healthcare outcomes of death rate, length of ICU stay (LOS), drug charges, et al.

\section{Methods}

Study Design and Participants

The study was performed in the medical-surgical ICU of the university-affiliated tertiary-care hospital with 20-bed in Beijing, China. The medical staff included board-certified intensivists, ICU fellows and residents. This study was a single-center, retrospective, cohort study using a pre-post design in the real world to evaluate the impact of the clinical pharmacist in attendance with ICU medical team on ICU patients' medication effectiveness and economics. The study was divided into two periods: a baseline period (from January 1, 2016 to October, 31, 2017) and an intervention period (from January 1, 2018 to October, $31,2019)$. The integrity of the patient is therefore not influenced by the intervention and all the data used in this study were collected anonymously.

\section{Pharmacist Practice}

The ICU clinical pharmacist, with more than 10 years of hospital practice experience, was assigned to the ICU ward to guarantee the continuity and quality of the intervention. This pharmacist is majored in antiinfectious diseases and she completed all the rotational training including 5 years of pharmacist resident training and 1 year of clinical pharmaceutical training on anti-infectious diseases. The pharmacist in the ICU focused on optimization of anti-infectious pharmacotherapy based on the professional guidelines or standards.

During the intervention period, the pharmacist attended daily unit rounds with ICU physicians at least 4 times a week and the average time invested by the ICU pharmacist was 4 hours per day. General responsibilities of the pharmacist in the ICU included comprehensive monitoring of medication usage such as indication, dose, frequency, solvent, curative effect, adverse drug reaction (ADR), inspection results and treatment course to assure the patients to obtain the proper and safety medication therapy. 
The full medication review was done using a standardized list. The pharmacist-identified medication related problems were then shared with the leader physician of treatment group. In general, pharmacists and physicians formed consensus after communication. The ward director would attend to discuss the discrepancy if it was necessary. Pharmacist consultative services in general pharmaceutical information, pharmacotherapy, or pharmacokinetics were also available as part of daily work in the ICU.

\section{Data collection}

All the patient information including the demography, infectious diagnosis of patients living in or transferred to ICU during 2016 to 2019 was abstracted from the Hospital Information of Intelligent Platform. Pharmaceutical service information was obtained from the ICU pharmacist daily records. The rate of consensus was defined as the percentage of recommendations agreed upon by the ICU physicians and the ICU pharmacist during the intervention period.

The primary outcome parameters were mortality in the ICU. The secondary outcome parameters were antibiotic AUD and utility rate, LOS, antibiotic charges and total drug charges. Routine antibiotic uses in ICU including carbapenems, broad-spectrum penicillin, third or fourth generation cephalosporins, monocyclic lactams, fluoroquinolones, aminoglycosides, tetracyclines, glycopeptides and oxazolidine and systemic antifungal agents. The antibiotic consumption was evaluated by antibiotic use density (AUD). AUD = Accumulative defined daily doses (DDDs) *100/ Length of stay of total patients in ICU (The number of patients who were treated the same period*Average days in hospital). DDDs $=$ Total drug consumption (g)/DDD. The standard DDD of antibiotics was confirmed on the website of World Health Organization $(\mathrm{WHO})^{[15]}$. AUD of each antibiotic category was calculated to compare the influence of ICU clinician's prescription tendency by the pharmacist.

\section{Statistical analysis}

Descriptive statistics were used to characterize the study group. Continuous variables were described as means \pm SDs or medians (interquartile range, IQR) according to the normal or non-normal distribution of the variables. Categorical variables were described as frequencies and percentage distributions. Data recorded for the pre-intervention (2016 to 2017) and intervention (2018 to 2019) periods were compared using the Pearson chi-square test or the Fisher exact test for categorical variables or the Student $t$ test or the Mann-Whitney $U$ test for quantitative variables according to the conditions of application. All data was analyzed using SPSS version 25.0 , and a P value of 0.05 or less was considered to indicate statistical significance.

\section{Results}

\section{Demographic characteristics of intervention patients}

The patients' basic characteristics before and after pharmacist attendance to the ICU were summarized in Table1. All the patients were admitted to the ICU by transfer from the emergency department or the other 
departments. There were no significant differences in baseline characteristics before and after the pharmacist attendance. The clinical pharmacist evaluated and completed the full anti-infectious medication reviews of the 1436 patients with infectious diseases. The median age of patients that intervened was 80 years old.

\section{Pharmaceutical recommendations and the consensus with the ICU clinicians}

In aggregate, of 1252 pharmacist-recommendations were identified, of which consensus between the ICU hospital pharmacist and the ICU clinicians was reached to 1169 (92.65\%). The main points that the pharmacist communicated with ICU clinicians were medication regimen adjustments (655/1252, $52.32 \%)$, drug withdrawal $(283 / 1252,22.60 \%)$, therapeutic drug monitoring (TDM) recommendations (218/1252, 17.41\%), dosage adjustment (82/1252, 6.55\%) and miscellaneous (14/1252, 1.12\%). Medication regimen adjustments got higher consensus rate $(626,95.57 \%)$ and discontinue drug presented the lower $(235,83.04 \%)$. The recommendations and the consensus were shown in table 2 .

The interventions were mainly conducted according to the professional anti-infectious guidelines (365/1252, 29.15\%), microbial susceptible test (253/1252, 20.21\%), TDM recommendations or results $(172 / 1252,13.74 \%)$ and drug withdrawal because of the full course of treatment $(167 / 1252,13.34 \%)$ or unnecessary drug utilities $(78 / 1252,6.23 \%)$. The corresponding consensus rate between the pharmacist and the clinicians was $96.71 \%, 94.07 \%, 97.67 \%, 77.84 \%$ or $87.18 \%$, respectively. Further details were shown in figure 1.

\section{The influence to ICU clinicians on drug selection tendency}

Since the microorganism detections could also affect the tendency of clinicians' prescription, we also collected the corresponding data. We found that though in our ICU, both of the microbial detection rate of gram-negative and positive bacterium were higher during the year of 2018 to 2019 than that during the year of 2016 to 2017 (8436/10871, 77.6\% vs 7590/10182, 74.54\% and 981/10871, 9.02\% vs $774 / 10182$, $7.60 \%$ ) and the fungi detection rate was the opposite (986/10871, $9.07 \%$ vs $1445 / 10182,14.19 \%)$, the antibiotics of AUD decreased significantly before and after pharmacist attendance to the ICU including the types of carbapenems $(p=0.000)$, broad-spectrum penicillin $(p=0.000)$, third and fourth generation cephalosporins $(p=0.012)$, fluoroquinolones $(p=0.000)$, aminoglycosides $(p=0.000)$, tetracyclines $(p=0.000)$, glycopeptides and oxazolidine $(p=0.000)$ and no decrease in the use of systemic antifungal agents $(p=0.445)$. On the whole, the AUD of all antimicrobials consumed in the ICU decreased from 211.83 to174.02 compared with pre- and post-pharmaceutical interventions $(p=0.000)$. The common microbial detections and AUD of each antibiotic category were shown in figure 2 and table 3.

\section{Clinical and economic outcomes with and without ICU pharmacists}

As shown in table 4, the rate of antimicrobial utility in ICU decreased from $89.88 \%$ to $86.82 \%$ after the clinical pharmacist participation and had statistical difference $(p=0.001)$. The mortality in ICU with pharmacist decreased compared to that without pharmacist (18.73\% vs $15.21 \%)$ and had statistical 
difference $(p=0.002)$. Moreover, the average length of stay (LOS) in ICU had no difference with or without pharmacist attendance $(9.07 \pm 9.47$ vs $8.96 \pm 11.46$ days, $p=0.755)$.

About the agent charges, pharmacist attendance potentially decreased the antibiotic charges from $8,644 \pm 12,556$ to $5,587 \pm 7,606(p=0.000)$ with $39 \%$ reduction. Correspondingly, all agent discharges were reduced from $26,023 \pm 31,787$ vs $20,160.17 \pm 24,309(p=0.000)$ with $27 \%$ reduction.

\section{Discussion}

Antibiotic exposure in intensive care patients with sepsis is frequently inadequate and is associated with poorer outcomes as critically ill patients have altered antibiotic pharmacokinetics. Clinically relevant pharmacokinetic knowledge on antibiotic dosing among intensive care professionals is insufficient[16]. This report describes the successful integration of a clinical pharmacist into ICU clinic and outlines the primary roles the pharmacist can serve within the ICU health care team, particularly in terms of cost avoidance outcomes.

There are three modes of collaboration between pharmacists and clinicians now, pharmacist-clinician cooperation, pharmacist-driven and clinician attendance or pharmacist alone. Different service target group and work target choose different work patterns. In the ICU, because of the complexity of the patients, the severity of the disease, and the diversity of drug use, multidisciplinary approach would be optimal[17]. In our ICU, the pharmacist works in the ward, reviews all the critical infectious patient medication regimens and exchanges views with the physician about any treatment issues found. Medication regimen adjustments, the timing of antimicrobial therapy withdrawal or TDM recommendation were the main points that the pharmacist interventions. The final treatment decision is based on full discussion and communication between the pharmacist and the physician, so the consensus rate was more than $90 \%$ or higher.

Pharmacist attendance can also realize the individualized medication evaluations and drug selections in the ICU. Carbapenems and glycopeptides/oxazolidine were the most applied in the ICU before. The timing of withdrawal, the choice of empirical use, the necessity of gram-positive coccal coverage, the misunderstanding of ideas are the common reasons for the excessive choice of these two types of drugs. Since each ICU physician is responsible for treating 4 to 6 critically ill patients per day, lot of clinic work made it impractical to require every physician to evaluate each patient's medication regimen individually. In addition, physicians are more willing to deal with diagnose or medically related treatments according to their specialties. Therefore, pharmacists can be a good medical supplement in the ICU. Our result data showed this very well.

The roles of the pharmacists on patient care had been proved by many studies[10,18-21]. Lee et al reported that including critical care pharmacists in the multidisciplinary ICU team improved patient outcomes including mortality, ICU length of stay in mixed ICUs, and preventable/non-preventable adverse drug events[22]. In our study, after pharmacist attendance, the rate of antimicrobial agent utilizations in the discharged patients decreased and patient antibiotic charges including the total drug costs were 
saving per stay. However, we pleasantly find that the ICU mortality did not increase, but rather decrease. LOS failed to shorten after pharmacist intervention, since this indicator was always be one of the important independent performance management indicators in clinic.

This study has several limitations. First, this intervention study was designed without involving a simultaneous control ICU group, so the results in this study may be probably influenced by the period. Second, this was a single-center study. Every medical setting has different cooperation patterns between clinical pharmacists and ICU physicians, as well as different work priorities, and then leading to different roles. Therefore, our results might not be extended to other ICU settings. Third, although mortality decreased significantly during the pharmacist activities, it might not only be attributed to the role of the pharmacist, but could also be related to the clinic management carried out in the ward during that time. Hence, more rigorous studies are needed to confirm the role of the ICU clinical pharmacists.

\section{Conclusions}

In summary, this study demonstrated that the services provided by clinical pharmacist with highly professional training in caring for critically ill patients with infections could optimize the antibiotic therapy regimes and significantly saved the drug costs. Our conclusion also indicated that at least the reduction of antibacterial feeding intervened by the pharmacist did not cause the critical infectious patients' under-treatment and then increase the mortalities.

These results need to be validated with further studies and the specific activities associated with the greatest benefit determined.

\section{Declarations}

\section{Funding}

The project described was supported by the Beijing Municipal Administration of Hospitals Incubating Program (Code PG2020014).

\section{Authors' contributions}

All authors provided a substantial contribution to the design and interpretation of the protocol, as well as writing or revising sections of drafts. Wei Chen designed the studies, wrote the protocol and revised the final manuscript. Hongyan Gu conducted the pharmacist interventions in the ward and wrote the manuscript. Lulu Sun wrote the protocol, conducted partial pharmaceutical interventions and revised the manuscript. Other authors coordinated the data extraction and processing.

\section{Acknowledgments}

Thanks for Electronic information retrieval support from Tian Zongmei, Wei Zhou, Zhou Yi, Li Jingyi and Yaqi Wang. They are all from Information center of Beijing Shijitan Hospital Affiliated to Capital Medical 
University, Beijing, China.

Competing interests

The authors declare no competing interests.

Ethics approval and consent to participate

The study was approved by the Scientific Ethics Committee, Beijing Shijitan Hospital Affiliated to Capital Medical University. All authors read and approved the final manuscript.

\section{Consent for publication}

Not applicable.

The data that support the findings of this study are available from the hospital information system from Beijing Shijitan Hospital, Beijing, China, but restrictions apply to the availability of these data, which were used under license for the current study, and so are not publicly available. Data are however available from the authors upon reasonable request and with permission of Beijing Shijitan Hospital.

\section{References}

1. Kollef MH: Optimizing antibiotic therapy in the intensive care unit setting. Crit Care 2001, 5(4):189195.

2. Kram BL, Trammel MA, Kram SJ, Wheeley SE, Mancheril BG, Burgess LD, Schultheis JM: Medication Histories in Critically III Patients Completed by Pharmacy Personnel. Ann Pharmacother 2019, 53(6):596-602.

3. Carson-Stevens A, Hingston CD, Wise MP: Minimising drug errors in critically ill patients. Crit Care 2011, 15(1):401.

4. Kollef $\mathrm{MH}$ : Antibiotics for the critically ill: more than just selecting appropriate initial therapy. Crit Care 2013, 17(3):146.

5. Warden BA, Shapiro MD, Fazio S: The Role of the Clinical Pharmacist in a Preventive Cardiology Practice. Ann Pharmacother 2019, 53(12):1214-1219.

6. American Pharmacists A: DOTx. MED: Pharmacist-delivered interventions to improve care for patients with diabetes. J Am Pharm Assoc (2003) 2012, 52(1):25-33.

7. Falamic S, Lucijanic M, Hadziabdic MO, Marusic S, Bacic Vrca V: Pharmacist's interventions improve time in therapeutic range of elderly rural patients on warfarin therapy: a randomized trial. Int $J$ Clin Pharm 2018, 40(5):1078-1085.

8. Montazeri M, Cook DJ: Impact of a clinical pharmacist in a multidisciplinary intensive care unit. Crit Care Med 1994, 22(6):1044-1048. 
9. Alvarez-Lerma F, Grau S, Echeverria-Esnal D, Martinez-Alonso M, Gracia-Arnillas MP, Horcajada JP, Masclans JR: A Before-and-After Study of the Effectiveness of an Antimicrobial Stewardship Program in Critical Care. Antimicrob Agents Chemother 2018, 62(4).

10. DiazGranados CA: Prospective audit for antimicrobial stewardship in intensive care: impact on resistance and clinical outcomes. Am J Infect Control 2012, 40(6):526-529.

11. Stollings JL, Bloom SL, Wang L, Ely EW, Jackson JC, Sevin CM: Critical Care Pharmacists and Medication Management in an ICU Recovery Center. Ann Pharmacother 2018, 52(8):713-723.

12. MacLaren R, Bond CA, Martin SJ, Fike D: Clinical and economic outcomes of involving pharmacists in the direct care of critically ill patients with infections. Crit Care Med 2008, 36(12):3184-3189.

13. Miyagawa $\mathrm{Cl}$, Rivera JO: Effect of pharmacist interventions on drug therapy costs in a surgical intensive-care unit. Am J Hosp Pharm 1986, 43(12):3008-3013.

14. Leape LL, Cullen DJ, Clapp MD, Burdick E, Demonaco HJ, Erickson JI, Bates DW: Pharmacist participation on physician rounds and adverse drug events in the intensive care unit. JAMA 1999, 282(3):267-270.

15. https://www.whocc.no/atc_ddd_index.

16. Fleuren LM, Roggeveen LF, Guo T, Waldauf P, van der Voort PHJ, Bosman RJ, Swart EL, Girbes ARJ, Elbers PWG: Clinically relevant pharmacokinetic knowledge on antibiotic dosing among intensive care professionals is insufficient: a cross-sectional study. Crit Care 2019, 23(1):185.

17. Ibrahim $\mathrm{KH}$, Gunderson B, Rotschafer JC: Intensive care unit antimicrobial resistance and the role of the pharmacist. Crit Care Med 2001, 29(4 Suppl):N108-113.

18. Fortin R: Role of the Pharmacist in Antimicrobial Stewardship. $R$ I Med J 2018, 101(5):26-27.

19. Kwak SH, Jeong CW, Lee SH, Lee HJ, Koh Y: Current status of intensive care units registered as critical care subspecialty training hospitals in Korea. Journal of Korean medical science 2014, 29(3):431-437.

20. MacLaren R, Bond CA: Effects of pharmacist participation in intensive care units on clinical and economic outcomes of critically ill patients with thromboembolic or infarction-related events. Pharmacotherapy 2009, 29(7):761-768.

21. Leguelinel-Blache G, Nguyen TL, Louart B, Poujol H, Lavigne JP, Roberts JA, Muller L, Kinowski JM, Roger C, Lefrant JY: Impact of Quality Bundle Enforcement by a Critical Care Pharmacist on Patient Outcome and Costs. Crit Care Med 2018, 46(2):199-207.

22. Lee H, Ryu K, Sohn Y, Kim J, Suh GY, Kim E: Impact on Patient Outcomes of Pharmacist Participation in Multidisciplinary Critical Care Teams: A Systematic Review and Meta-Analysis. Crit Care Med 2019, 47(9):1243-1250.

\section{Tables}

Tab 1 Baseline Characteristics of patients before or after pharmacist attendance to the ICU 


\begin{tabular}{|c|c|c|c|}
\hline Characteristic & $\begin{array}{l}\text { Before pharmacist attendance } \\
\square N=1242 \square\end{array}$ & $\begin{array}{l}\text { After pharmacist attendance } \\
\square N=1436 \square\end{array}$ & p \\
\hline Gender & & & 0.112 \\
\hline Male & 704 (56.68\%) & 770 (53.62\%) & \\
\hline Age & & & 0.292 \\
\hline Median (years, IQR) & $83(70-88)$ & $80(67-87)$ & \\
\hline$<65 y$ & 213 (17.15\%) & 301 (20.96\%) & \\
\hline $65 \sim 85 y$ & $561(45.17 \%)$ & 709 (49.37\%) & \\
\hline$\geq 86 y$ & $468(37.68 \%)$ & $426(29.67 \%)$ & \\
\hline Admission times & & & 0.561 \\
\hline 1 & 998 (80.55\%) & 1200 (83.57\%) & \\
\hline $2 \sim 4$ & $171(13.80 \%)$ & $183(12.74 \%)$ & \\
\hline$\geq 5$ & 70 (5.65\%) & 53 (3.69\%) & \\
\hline 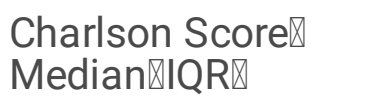 & $5 \rrbracket 3 \sim 6 \rrbracket$ & $4 \otimes 2 \sim 5 \rrbracket$ & 0.878 \\
\hline
\end{tabular}

Tab 2 Practices promoting the optimization of antimicrobial use in the ICU

\begin{tabular}{|c|c|c|}
\hline Type of intervention & Intervention Number $₫$ Proportion $\otimes \%)$ & Consensus (Rate, \%) \\
\hline Medication regimen adjustment & $655 \rrbracket 52.32 \% \rrbracket$ & $626(95.57 \%)$ \\
\hline Drug withdrawal & $283 \rrbracket 22.60 \% \bigotimes$ & $235(83.04 \%)$ \\
\hline Lab or TDM recommendation & $218 \otimes 17.41 \% \bigotimes$ & $213(97.71 \%)$ \\
\hline Dosage adjustment & $82 \bigotimes 6.55 \% \rrbracket$ & $74(90.24 \%)$ \\
\hline Miscellaneous & $14 \rrbracket 1.12 \% \rrbracket$ & $12(85.71 \%)$ \\
\hline Total & 1252 & $1169(92.65 \%)$ \\
\hline
\end{tabular}

Tab 3 The AUD influence of the ICU clinicians on drug selection tendency 


\begin{tabular}{|lllll|}
\hline & $\begin{array}{l}\text { Before pharmacist } \\
\text { attendance }\end{array}$ & $\begin{array}{l}\text { After pharmacist } \\
\text { attendance }\end{array}$ & Reduction[\% & p \\
\hline Carbapenems & 45.98 & 24.99 & $45.66 \%$ & $0.000^{*}$ \\
\hline Broad-spectrum penicillin & 7.74 & 12.88 & $-66.40 \%$ & $0.000^{*}$ \\
\hline $\begin{array}{l}\text { Third and fourth generation } \\
\text { Cephalosporins }\end{array}$ & 15.82 & 8.71 & $44.98 \%$ & $0.012^{*}$ \\
\hline Monocyclic lactams & 9.39 & 19.44 & $-106.95 \%$ & 0.898 \\
\hline Fluoroquinolones & 6.44 & 7.67 & $-19.05 \%$ & $0.000^{*}$ \\
\hline Aminoglycosides & 21.79 & 19.53 & $10.37 \%$ & $0.000^{*}$ \\
\hline Ttetracyclines & 19.44 & 12.14 & $37.52 \%$ & $0.000^{*}$ \\
\hline $\begin{array}{l}\text { Glycopeptides and } \\
\text { Oxazolidine }\end{array}$ & 40.52 & 28.12 & $30.60 \%$ & $0.000^{*}$ \\
\hline Systemic antifungal agents & 39.38 & 33.22 & $15.62 \%$ & 0.445 \\
\hline Total AUD & 211.83 & 174.02 & $17.85 \%$ & $0.000^{*}$ \\
\hline
\end{tabular}

Tab. 4 Clinical and economic outcomes of critically ill patients with infectious diseases with and without clinical pharmacists in ICUs.

\begin{tabular}{|c|c|c|c|}
\hline Clinical and economic outcomes & $\begin{array}{l}\text { Before pharmacist } \\
\text { attendance }\end{array}$ & $\begin{array}{l}\text { After pharmacist } \\
\text { attendance }\end{array}$ & $\mathbf{P}$ \\
\hline Mortality, No. (\%) & $391 \otimes 18.73 \% \rrbracket$ & $356 \rrbracket 15.21 \% \rrbracket$ & $0.002^{*}$ \\
\hline $\begin{array}{l}\text { Cases number of antimicrobial agent } \\
\text { utilizations in the discharged patients (\%) }\end{array}$ & $\begin{array}{l}2176 / 2421 \\
89.88 \%\end{array}$ & $\begin{array}{l}2358 / 2716 \\
86.82 \%\end{array}$ & $0.001 *$ \\
\hline LOS, (Means days \pm SD) & $8.96 \pm 11.46$ & $9.09 \pm 9.47$ & 0.755 \\
\hline $\begin{array}{l}\text { Antibiotic charges per stay ( } \nabla / \text { case) (Means } \\
\pm S D \text { ) }\end{array}$ & $8,644 \pm 12,556$ & $5,587 \pm 7,606$ & $0.000 *$ \\
\hline Drug charges per stay $\mathbb{\mathbb { Z }} /$ case $\mathbb{\mathbb { }}$ (Means $\pm \mathrm{SD}$ ) & $26,023 \pm 31,787$ & $20,160.17 \pm 24,309$ & $0.000 *$ \\
\hline Laboratory charges (\$/case) & & & \\
\hline
\end{tabular}

\section{Figures}


Fig 1 Further categories of pharmacist interventions

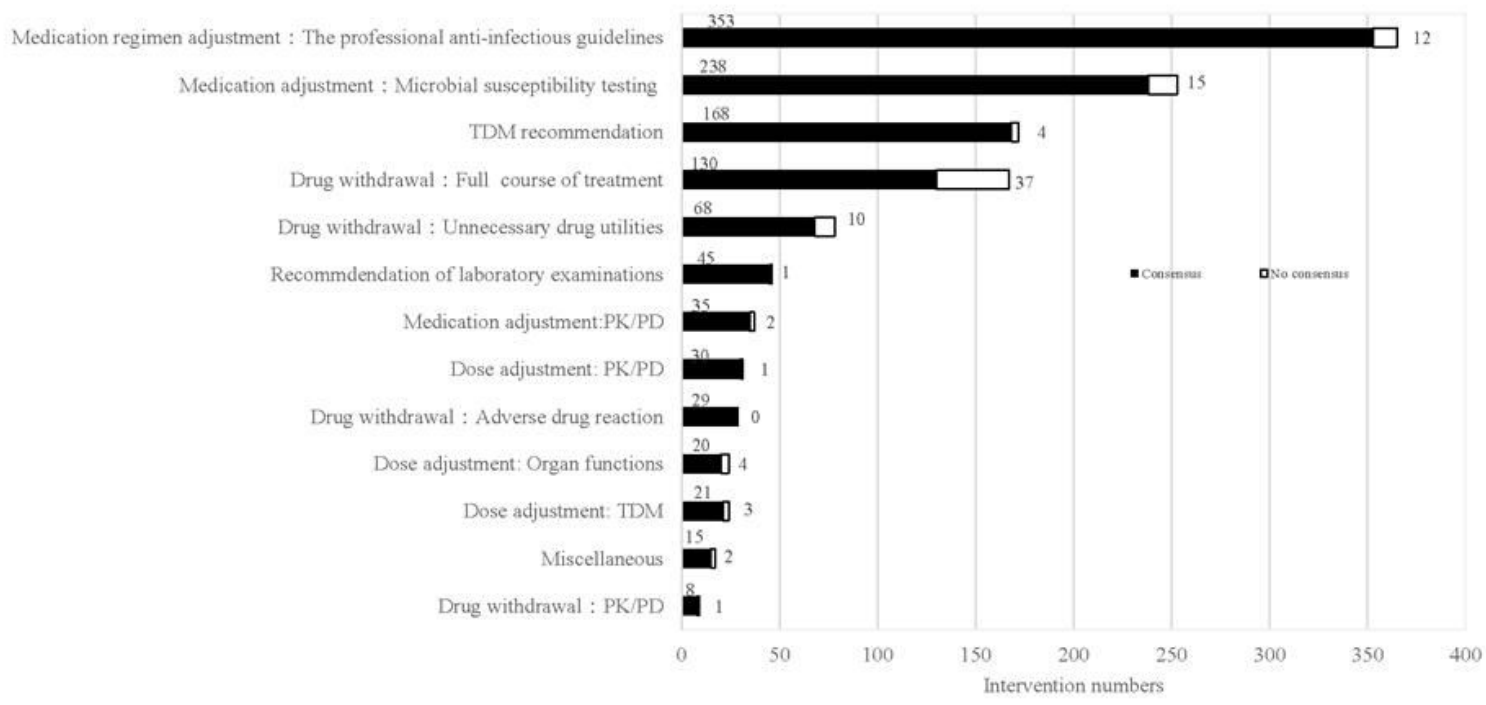

\section{Figure 1}

\section{Further categories of pharmacist interventions}

Fig 2 The proportion of microbial detections before and after pharmacist attendance

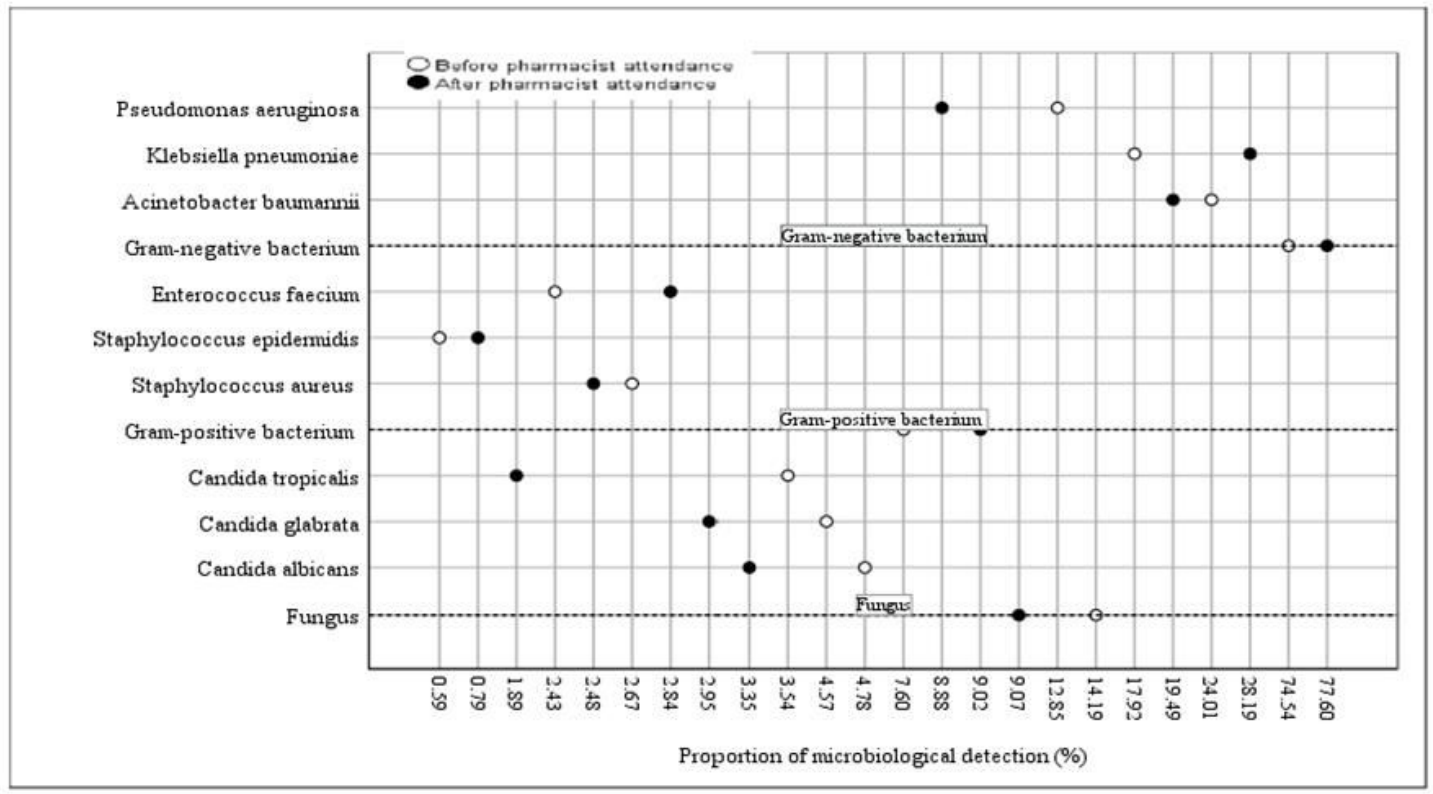


Figure 2

The proportion of microbial detections before and after pharmacist attendance 